\title{
Celos y violencia en parejas de estudiantes de la Universidad de Cuenca, Ecuador
}

\section{Jealousy and intimate partner violence in students of the University of Cuenca, Ecuador}

\section{Ciúmes e violência em casais de estudantes da Universidade de Cuenca, Equador}

\author{
Ximena Guillén Verdesoto1 ORCID 0000-0003-0690-7941 \\ Juana Ochoa Balarezo² ORCID 0000-0001-5683-6657 \\ Gustavo Delucchi ${ }^{3}$ ORCID 0000-0003-2771-1980 \\ Elizabeth León Mayer ${ }^{4}$ ORCID 0000-0003-1564-6448 \\ Jorge Folino 5 ORCID 0000-0001-5834-2212 \\ ${ }^{12}$ Universidad de Cuenca. Ecuador \\ ${ }^{345}$ Facultad de Ciencias Médicas, Universidad Nacional de La Plata. Argentina
}

\begin{abstract}
Resumen: En esta investigación se dimensiona la violencia en parejas de estudiantes universitarios y se explora su relación con los celos y otros correlatos. Con un diseño de corte transversal se encuestaron 186 estudiantes de Universidad de Cuenca. Los instrumentos utilizados fueron el Conflict in Adolescents Dating Relationships Inventory, la Multidimensional Jealousy Scale e items ad hoc sobre fidelidad, consumo de sustancias y estrés durante la relación de pareja. Los puntajes de violencia fueron bajos en general, pero preocupantes en el 5.4\% de los encuestados. El 60.2\% informó haber cometido por lo menos un acto violento físico durante la relación de pareja index. Los subtipos de violencia medidos tuvieron distribución similar entre varones y mujeres. La correlación entre violencia total cometida y sufrida fue $.77(p<.001)$. Se verificó asociación significativa entre la pertenencia al grupo de mayor violencia y los celos comportamentales y el estrés padecido durante la pareja.
\end{abstract}

Palabras Clave: violencia de pareja, estudiante universitario, celos, fidelidad, estrés, consumo de sustancias

Abstract: This research work evaluates violence in couples of university students and focuses on their relationship with jealousy and other variables. Using a cross-sectional design, 186 students from the University of Cuenca, Ecuador, were surveyed. Instruments used were Conflict in Adolescents Dating Relationships Inventory, Multidimensional Jealousy Scale, and other ad hoc methods related to fidelity, substance use, and stress during dating relationship. Violence scores observed were low in general though disturbing in 5.4\% of students surveyed. Noticeably, $60.2 \%$ of them reported to have committed at least one physical violent act during their index dating relationship. Violence subtypes measured showed similar distribution between men and women. Correlation between total violence committed and suffered was $.77(p<.001)$. A significant association was observed between the highest violence group and behavioral jealousy and stress during dating relationship. Key Words: partner violence, university student, jealousy, fidelity, stress, substance use 
Resumo: Nesta pesquisa, dimensiona-se a violência em casais de estudantes universitários e explora-se sua relação com o ciúme e outros correlatos. Com um desenho transversal, foram estudados 186 alunos da Universidade de Cuenca. Os instrumentos utilizados foram o Conflict in Adolescents Dating Relationships Inventory, a Multidimensional Jealousy Scale e itens ad hoc sobre fidelidade, uso de substâncias e estresse no relacionamento. Os escores de violência foram baixos em geral, mas preocupantes em 5,4\% dos participantes. 60,2\% relataram ter cometido pelo menos um ato de violência física durante o relacionamento. Os subtipos de violência medidos tiveram distribuição semelhante entre homens e mulheres. A correlação entre o total de violência cometida e sofrida foi de .77 ( $\mathrm{p}<.001$ ). Houve associação significativa entre pertencimento ao grupo de maior violência, ciúme comportamental e estresse sofrido durante o relacionamento.

Palavras-chave: violência de casal, estudante universitário, ciúmes, fidelidade, estresse, uso de substâncias

Recibido: 23/03/2020

Aceptado: 29/10/2020

Cómo citar:

Guillén Verdesoto, X., Ochoa Balarezo, J., Delucchi, G., León Mayer, E., \& Folino, J. (2021). Celos y violencia en parejas de estudiantes de la Universidad de Cuenca, Ecuador. Ciencias Psicológicas, 15(1), e-2353. doi: https://doi.org/10.22235/cp.v15i1.2353

Correspondencia: Ximena Guillen. Coordinadora de Vinculación con la Sociedad de la Facultad de Jurisprudencia y Ciencias Políticas y Sociales de la Universidad de Cuenca. Ecuador. E-mail:ximena.guillen@ucuenca.edu.e

La violencia en la pareja se manifiesta con diversas exteriorizaciones y diversos grados de letalidad (Organización Panamericana de la Salud, 2003). La adolescencia tardía y la adultez temprana han sido señalados como grupos etarios de mayor riesgo (Capaldi, Knoble, Shortt, \& Kim, 2012). En esta investigación se focaliza sobre la violencia en las parejas de estudiantes universitarios (en adelante VPEU) y se indaga sobre la magnitud del fenómeno, sus diferentes formas de presentación y su relación con los celos y otras covariables en el contexto de una institución ecuatoriana.

\section{La Violencia de Pareja}

Diversos estudios han puesto de manifiesto que haber tenido la experiencia de algún tipo de violencia en la pareja es común en muy diversos grupos étnicos o sociales. Por ejemplo, Chang, Shen, y Takeuchi (2009) hallaron que $10.2 \%$ de las mujeres y $12 \%$ de los varones de una muestra representativa de la población asiático-americana en los EEUU informaron haber sido víctimas de violencia por parte de sus actuales parejas. En un reciente meta-análisis sobre una población combinada de 329.212 militares de Canadá y EEUU se halló que la prevalencia de violencia física cometida en el último año fue del $26 \%$ en los varones y del $20 \%$ en las mujeres (Kwan et al., 2020). Otros estudios informaron prevalencias del padecimiento de la violencia de pareja en la vida del $24.3 \%$ para mujeres y del $13.8 \%$ para varones (Black et al., 2010).

En lo que respecta a la violencia de pareja en adolescentes se cuenta con información temprana que daba cuenta de un rango de prevalencia entre el 9\% y el 57\% (Cascardi, AveryLeaf, \& O’Leary, 1994; Roscoe \& Callahan, 1985). Posteriormente, el Centro de Control de 
Enfermedades reportó que el 12\% de estudiantes secundarios habían padecido violencia física en una relación de pareja (Center for Disease Control and Prevention, 2000). La alta prevalencia de la violencia entre jóvenes estudiantes en noviazgo también fue ampliamente documentada en un estudio internacional que utilizó muestras de 22 países (Chan, Straus, Brownridge, Tiwari, \& Leung, 2008). El estudio no incluyó a Ecuador pero sí lo hizo con otros dos países latinoamericanos (México y Brasil); los resultados del país ubicado en la mediana indicaron que el $30 \%$ de los estudiantes de la muestra informaron haber cometido agresión física a su pareja en los previos 12 meses (Chan et al., 2008).

Por otra parte, también se cuenta con evidencias que ponen de manifiesto una tendencia a que concurran, en las relaciones adolescentes, la condición de víctima de violencia con la de perpetrador (Chan et al., 2008; Malik, Sorenson, \& Aneshensel, 1997; Straus \& Ramirez, 2007; Taylor \& Mumford, 2016).

La violencia en la pareja puede manifestarse de diversas maneras. Burk y Seiffge-Krenke (2015) agrupan las manifestaciones violentas en dos tipos: la agresión física y la agresión relacional. La primera consiste en el uso intencional de la fuerza que puede herir a la pareja y que incluye tanto conductas agresivas leves -por ejemplo, empujones, rasguños- como conductas violentas severas -por ejemplo, intentos de asfixia, bofetadas, ataques con armas-. La segunda involucra insultos, ataques verbales, chismes difamatorios, exclusión, coqueteo sutil con otra persona con la intención de producir celos y amenazas de terminar la relación. Esta forma de agresión relacional se asocia con mala adaptación psicológica, depresión, y una mala calidad de relación (Prinstein, Boegers, \& Vernberg, 2001). Las mujeres, comparativamente con los varones, tienden a molestarse más por ese tipo de agresión relacional, perciben que tiene un mayor impacto en sus relaciones, y pasan más tiempo pensando y discutiendo acerca de ello (Salmivalli \& Kaukiainen, 2004). Cuando la violencia en las parejas es mutua se acompaña de funcionamiento menos adaptativo, más conflictos y carencia de cualidades relevantes como son la confianza y el sostén (Seiffge-Krenke \& Burk, 2015).

Malik et al. (1997) informaron que las adolescentes de colegios de Los Ángeles tuvieron mayor probabilidad que los varones de comportarse violentamente durante el noviazgo y que entre los factores que aumentaron el riesgo se contaba la exposición a previa violencia, la normalización del uso de la violencia y el consumo de marihuana. Capaldi et al. (2012) realizaron una revisión sistemática y entre los factores de riesgos demográficos, encontraron que a mayor edad disminuye el riesgo de violencia de pareja y que el pico de la misma se ubica en la adolescencia tardía o adultez temprana. Johnson, Giordano, Manning, y Longmore (2014) adicionaron el detalle que en los varones la violencia en la pareja aumenta desde los 13 hasta los 20 años y luego decrece y que para las mujeres, si bien el patrón es similar, alcanza el pico entre $\operatorname{los} 21$ y 24 años.

Los estudios del medio latinoamericano dan cuenta también de las altas prevalencias y de la simetría en la violencia durante los noviazgos. En un estudio en 963 estudiantes de la universidad pública de Córdoba, Argentina, se halló que la prevalencia de agresión física en los últimos 12 meses fue del $34 \%$ para las mujeres y del $22 \%$ para los varones, y que la manifestación de haber cometido agresiones incrementó significativamente la probabilidad de sufrir agresiones de la misma modalidad o de otra (Arbach-Lucioni, Nguyen-Vo, \& Bobbio, 2015). En otro estudio argentino se informó que la VPEU en una muestra de 528 estudiantes de la Facultad de Ciencias Médicas de la Universidad Nacional de La Plata, tuvo una media de 0.35 $(D E=0.27$; rango posible $0-3)$; que la forma de violencia predominante fue la verbal emocional $(M=0.85 ; D E=0.5)$; que las puntuaciones se concentraban en los valores más bajos pero que el $3.2 \%$ tuvo preocupantes valores mayores a 1 en el total de violencia cometida; que el $50.2 \%$ del total, el $19.1 \%$ de las mujeres y el $20.6 \%$ de los varones había informado haber cometido, por lo menos, un acto violento físico durante la relación índex (Delucchi, Leon-Mayer, \& Folino, 2020). 


\section{Los Celos y la Violencia de Pareja}

La literatura científica ofrece diversas evidencias que señalan la influencia de los celos en la violencia de pareja. Los celos y la violencia del varón hacia la pareja mujer han sido tempranamente conceptualizados como parte de una propensión psicológica que evolucionó en el Homo Sapiens con la funcionalidad de alcanzar confianza en la paternidad y de asegurar la exclusividad y control sexual de la mujer (Daly, Wilson, \& Weghorst, 1982). Más recientemente, la atención se movilizó hacia el estudio de la relación entre celos y otros constructos de nivel individual con la agresión u otras consecuencias problemáticas y dejó de restringirse al sexo masculino y a la funcionalidad para la reproducción, para interesarse, también, en jóvenes transgénero y jóvenes no conformistas (Goldenberg, Jadwin-cakmak, \& Harper, 2018).

Los celos se relacionaron con la agresión psicológica y física en jóvenes norteamericanos (Davis, Ace, \& Andra, 2000; Giordano, Soto, Manning, \& Longmore, 2010), y en estudiantes españoles (Muñoz-Rivas, Graña, O'Leary, \& González, 2007). La intensidad de los celos, mediados por amenaza a la autoestima, se relacionó con la provocación de la agresión hacia la pareja en estudio experimental en Boston (DeSteno, Valdesolo, \& Bartlett, 2006).

Murphy y Russell (2016) hallaron que los celos resultaron mediadores de la relación entre la sensibilidad al rechazo y la agresión y DiBello, Rodriguez, Hadden, y Neighbors (2015), que los celos cognitivos mediaron en la asociación entre la autoestima contingente a la relación de pareja, el consumo de alcohol y los problemas relacionados con ese consumo.

En el medio latinoamericano, un aporte desde la investigación cualitativa feminista puso de manifiesto la relación entre los celos, la infidelidad y el consumo de sustancias con los conflictos y la violencia de pareja (do Nascimento Paixao et al., 2014). En el mismo país, un estudio cuantitativo encontró significativa asociación entre celos patológicos no delirantes y el rasgo de ansiedad, asociación que podría aumentar la reactividad violenta (Costa, Sophia, Sanches, Tavares, \& Zilberman, 2015). De la Argentina se cuenta con los hallazgos de la investigación antes mencionada que orientan a sostener la existencia de relación significativa entre los celos y la violencia de pareja (Delucchi, Leon-Mayer, \& Folino, 2018). Específicamente, en ese estudio se encontró que los celos comportamentales y los celos cognitivos se asociaron positiva y significativamente con la VPEU, aun controlando otros factores tales como el estrés y el consumo de sustancias durante la relación de pareja y la edad al inicio de esa relación (Delucchi et al., 2020).

\section{Infidelidad, consumo de sustancias, estrés y violencia de pareja}

Los celos, la infidelidad de la pareja y el uso de alcohol y drogas son situaciones que tienen relación con la construcción de la violencia conyugal (Paixão et al., 2014). Tempranas evidencias orientaron a considerar que la previa infidelidad, ya sea cometida o sufrida, influye aumentando la predisposición a reaccionar con celos (Pines \& Aronson, 1983). Más recientemente, se puso de manifiesto que haber sufrido infidelidad es un predictor significativo de los celos, especialmente en los varones (Burchell \& Ward, 2011) y que los varones despliegan sentimientos de ira y propensión hacia la violencia en respuesta a la infidelidad de la pareja (Miller \& Maner, 2008).

El consumo de sustancias es un factor asociado con riesgo de violencia de acuerdo a evidencias obtenidas en ámbitos comunitarios y asistenciales (Swanson, Holzer, Ganju, \& Jono, 1990; Swanson, Monahan, \& Steadman, 1994) y, también, en la relación de pareja (Capaldi et al., 2012). Brem, Shorey, Rothman, Temple, y Stuart (2018) encontraron al consumo de alcohol significativamente relacionado con la violencia de pareja en varones celosos. Ihongbe y Masho (2018) informaron que el consumo de marihuana sintética se asoció con mayor riesgo de victimización por violencia en los noviazgos juveniles. Si bien el consumo de sustancias interactúa con otras variables contextuales en el ámbito de la violencia de pareja juvenil, como 
son el sostén familiar y las expectativas estudiantiles (Gomez, Orchowski, Pearlman, \& Zlotnick, 2019), su exploración es relevante pues resulta un blanco de acciones preventivas.

El estrés es un componente reconocido de la diátesis hacia la violencia y tiene su lugar entre los factores que se contemplan al evaluar el riesgo de violencia (Douglas, Hart, Webster, \& Belfrage, 2013; Greer, Taylor, Cella, Stott, \& Wykes, 2020). En los noviazgos juveniles se lo considera una consecuencia directa de la violencia, o indirecta, a través del sufrimiento que, en ocasiones, produce el rechazo social hacia los jóvenes de parejas violentas (Temple et al., 2016). Recientemente se informaron evidencias que dan cuenta de una contribución significativa e independiente del estrés sobre la violencia de pareja (Buunk \& Massar, 2019).

\section{El Contexto}

El contexto de la investigación está configurado por la Universidad de Cuenca, ubicada en la ciudad de Cuenca, Ecuador. Se trata de una institución pública que tiene alrededor de 18.000 estudiantes en programas de pre y post grado. El estudio es pertinente a la política de la universidad pues ésta promueve el desarrollo de programas de atención y orientación a los estudiantes campañas de prevención contra las drogas y violencia, y de asistencia en casos de vulneración de derechos (Aula de Derechos Humanos, 2018; Consejo Universitario, 2018).

La determinación institucional para prevenir la violencia indica la pertinencia de promover la investigación empírica sobre los problemas de violencia de pareja en los estudiantes universitarios y desplegar acciones preventivas basadas en evidencias. Sin embargo, la indagación sistemática en el área es aún incipiente. Aunque sin poder parangonarse con el presente estudio por aspectos metodológicos, reciente información a nivel nacional da cuenta de la magnitud del problema en Ecuador al poner de relieve que, a lo largo de la vida, el $40.8 \%$ de mujeres vivieron violencia psicológica y una de cada cuatro, violencia física (Instituto Nacional de Estadísticas y Censos, 2019).

\section{El Presente Estudio}

El presente estudio tiene la meta de contribuir a la obtención de información que pueda servir de insumo para el establecimiento de programa de prevención de VPEU. Específicamente, el estudio tiene los objetivos de describir las manifestaciones violentas y de explorar su relación con los celos y otras variables teóricamente relevantes en una muestra de estudiantes universitarios de la Universidad de Cuenca. Operacionalmente, se plantea la hipótesis de una asociación positiva y significativa entre celos, estrés y consumo de sustancias y la VPEU.

\section{Materiales y método}

\section{Diseño y Participantes}

Con un diseño de corte transversal, se encuestaron estudiantes de las carreras Trabajo Social y Orientación Familiar de la Facultad de Jurisprudencia y Ciencias Políticas y Sociales de la Universidad de Cuenca entre los meses de abril y mayo de 2018. De 220 estudiantes que durante ese período estuvieron cursando asignaturas de Trabajo Social y de Orientación Familiar, pudieron ser encuestados 186: 150 mujeres $(80.6 \%)$, 35 varones $(18,8 \%)$ y un participante que no registró su sexo $(0.5 \%)$. La edad media al momento de la encuesta fue 22 años (DE 2.9; mín. 17; máx. 31).

\section{Instrumentos}

Conflict in Adolescents Dating Relationships Inventory -CADRI-. Se utilizó la versión de CADRI (Wolfe, 2001; Wolfe et al., 2001) traducida al español por Fernández Fuertes, Fuertes Martín, y Fernández Pulido (2006), con mínima adaptación a la terminología argentina 
(Delucchi et al., 2018). La CADRI está formada por 35 pares de ítems, que evalúan la presencia de conflicto y violencia en la pareja. Los ítems son enunciados de manera objetiva y comportamental (por ejemplo, "Le hablé con un tono hostil u ofensivo") y son contextualizados al momento del conflicto o pelea con la pareja. Los ítems se agrupan de a pares porque se pregunta por la conducta de quien responde y por la de su pareja. Veinticinco de los ítems son considerados para el cálculo del total y de las subescalas. La puntuación de cada ítem va desde 0 a 3 según un ordenamiento de frecuencias -0 corresponde a "nunca", 1 a "raramente o una o dos veces", 2 corresponde a "a veces o de 3 a 5 veces" y 3 a "a menudo o 6 o más veces". Los 10 pares de ítems restantes están relacionados con tácticas de resolución positiva de conflictos y cumplen rol de distractores y no forman parte de las mensuras. Los ítems de la CADRI se agrupan en cinco subescalas que representan respectivos dominios de la violencia de pareja: Violencia sexual" -cuatro ítems-; "Violencia relacional" - tres ítems-; "Violencia verbalemocional" -diez ítems-; "Amenazas" - cuatro ítems-; "Violencia física" -cuatro ítems-. Los ítems de la Violencia relacional hacen referencia a acciones tendientes a desacreditar a la pareja entre sus compañeros/amigos, mientras que los de la Violencia verbal-emocional hacen referencia a diversos tipos de insultos, amenazas, reproches o actos hechos a la pareja tendientes a hacerla enojar o a darle celos. Para cada subescala se calcula la media. En las restantes subescalas el dominio medido está literalmente representado por sus respectivas denominaciones. Estas subescalas contribuyen a formar dos puntajes de segundo orden: el de "Total de abuso cometido" y el de "Total de abuso sufrido" que se obtienen promediando las subescalas.

Los autores del instrumento original informaron los siguientes coeficientes alfa: total abuso cometido, .83; violencia sexual, .51; violencia relacional, .52; violencia verbal-emocional, .82; amenazas, .66 y violencia física, .83 (Wolfe, 2001; Wolfe et al., 2001). En reciente estudio argentino, los coeficientes Alfa obtenidos fueron .84, $.42, .51, .81, .82$ y .72 , respectivamente (Delucchi et al., 2020). Los valores en el presente estudio fueron .84, .35, .29, .79, .51, у .78 respectivamente. Wolfe et al. encontraron que las subescalas de abuso sexual y de agresión relacional variaban en su relación con el factor de segundo orden según edades y sexos, por lo que recomiendan utilizar como indicador general de violencia sólo la suma de las restantes tres subescalas (Wolfe, 2001; Wolfe et al., 2001). Siguiendo tal recomendación y considerando los coeficientes alfa, en el presente trabajo no se informan los resultados con las subescalas de abuso sexual y de agresión relacional y la información del Total de abuso cometido se refiere a la suma de las escalas Violencia verbal-emocional, Amenazas y Violencia física.

Multidimensional Jealousy Scale -MJS-. Para medir el constructo de celos se utilizó la MJS (Pfeiffer \& Wong, 1989) en su versión argentina (Delucchi et al., 2018). El instrumento fue desarrollado para evaluar los aspectos cognitivos, emocionales y conductuales de los celos en relaciones románticas. Diversos estudios informan buenos índices de consistencia interna superiores a .80 (Elphinston, Feeney, \& Noller, 2011; Haas Bueno \& Carvalho, 2005; Lucas, Pereira, \& Esgalhado, 2012; Pfeiffer \& Wong, 1989; Tani \& Ponti, 2016); en Argentina se obtuvieron valores de coeficiente Alfa de .90, .86, .88 y .88 para las subescalas Cognitiva; Emocional; Comportamental y para el total, respectivamente (Delucchi et al., 2020). Para la muestra del presente estudio, los valores fueron $.92, .87, .93$ y .87, respectivamente.

La escala está formada por 24 ítems que se distribuyen en tres factores: cognitivo, que es indicador de la frecuencia de los pensamientos relacionados con los celos; emocional que es indicador de la intensidad de la respuesta emocional o sentimiento; y conductual que es indicador de la frecuencia de las acciones relacionadas a los celos. La puntuación de cada ítem va de 1 a 7 que corresponden, respectivamente, a las siguientes categorías ordenadas: "todo el tiempo"; "casi siempre"; "muchas veces"; "la mitad del tiempo"; "pocas veces"; "casi nunca"; "nunca". Los valores de la subescala cognitiva deben ser revertidos antes de ser sumados. Las puntuaciones posibles en cada subescala tienen un rango de 8 a 56; el total tiene un rango de 24 a 
168 y los valores más altos son indicativos de los mayores niveles de celos (Pfeiffer \& Wong, 1989).

Escalas de fidelidad y de estrés. La valoración del encuestado sobre su propio patrón de fidelidad y sobre la fidelidad de previas parejas se midió con una escala cuyo rango va desde 0 (nada infiel) hasta 10 (completamente infiel). De la misma manera se midió la autovaloración del nivel de estrés del encuestado contextualizado en la época de la pareja índex, sin que se preguntara si el estrés era producido por conflictos de pareja u otra causa externa.

Level 2 -Substance use- adult. El consumo de sustancias contextualizado a la época de la pareja índex se midió en base a una de las escalas de medidas emergentes del DSM5. La versión en español se denomina NIVEL 2-Uso de sustancias-Adultos y se trata de un instrumento adaptado del ASSIST (Organización Mundial de la Salud, 2011) modificado por el National Institute on Drug Abuse (American Psychiatric Association, 2013). Si bien no se encontró información sobre la confiabilidad de la versión modificada en población universitaria, Mostardinha, Bártolo, Bonifácio, y Pereira (2019) informaron un rango de .56 a .84 en los coeficientes Alfa de las 10 subescalas de la versión original para esa población específica.

\section{Procedimiento}

Los ítems de los instrumentos y los diseñados ad hoc para obtener información demográfica se dispusieron en una encuesta en formato papel con las respectivas instrucciones. Esas instrucciones también se transmitieron verbalmente a los participantes junto con algunos contenidos pertinentes a la metodología. Posteriormente, se abrió oportunidad de dialogar para aclarar dudas.

Se solicitó a los participantes que contextualizaran las respuestas seleccionando una relación de pareja romántica intensa, actual o pasada, independiente del sexo y género de cualquiera de los miembros. La pareja sobre la que los encuestados respondieron, se denomina pareja índex en el resto del artículo.

A los estudiantes se les solicitó el consentimiento verbal luego de explicarles los objetivos de la investigación y detalles del procedimiento. Se tuvo el cuidado bioético de informárseles que la decisión de participar o no, de ninguna manera tendría impacto en la valoración de su desempeño estudiantil. También se les informó que la encuesta era anónima, que los resultados se expresarían estadísticamente y se pondrían a disposición de la comunidad científica y estudiantil. Además, se informó que el proyecto contaba con la autorización de la máxima autoridad universitaria.

\section{Análisis Estadístico}

Los datos se cargaron en base de datos con doble control y el análisis se realizó con SPSS 20.1. En primer lugar, se realizó el análisis descriptivo de las variables según su nivel de medición. Posteriormente, habiendo establecido un nivel de confianza de $p<.05$ se hizo el análisis bivariado con pruebas no paramétricas - prueba de Mann-Whitney y Rho de Spermandado que la amplia mayoría de las variables tuvieron distribuciones diferentes a normal según prueba de Kolmogorov-Smirnov. Finalmente se formuló un modelo de regresión logística con procedimiento de pasos hacia adelante según el test de Wald, con criterio de ingreso $p<.05$ y de salida $p<.10$. 


\section{Resultados}

Los encuestados brindaron la información sobre la pareja índex que había comenzado, en promedio, a los 18 años ( $D E 2.74$ años; mín. 13; máx. 26) y que tuvo una duración media de 33 meses ( $D E$ 30; mín 1; máx 144). El 49\% de los encuestados informó que aún continuaba con la relación al momento de la colección de datos.

La distribución de los valores de VPEU según CADRI difirió significativamente de la normal en todas las subescalas y en el total. La distribución de valores del total de abuso cometido según CADRI se expone en el Gráfico 1. El gráfico tiene en su abscisa el rango posible de la puntuación. Si bien la concentración de puntuaciones se ubica en los valores más bajos, se destaca que el 5.4\% tuvo valor de 1 o más en Abuso total cometido, lo que implica preocupante nivel de violencia. El $60.2 \%$ informó haber cometido, por lo menos, un acto violento físico durante la relación índex.

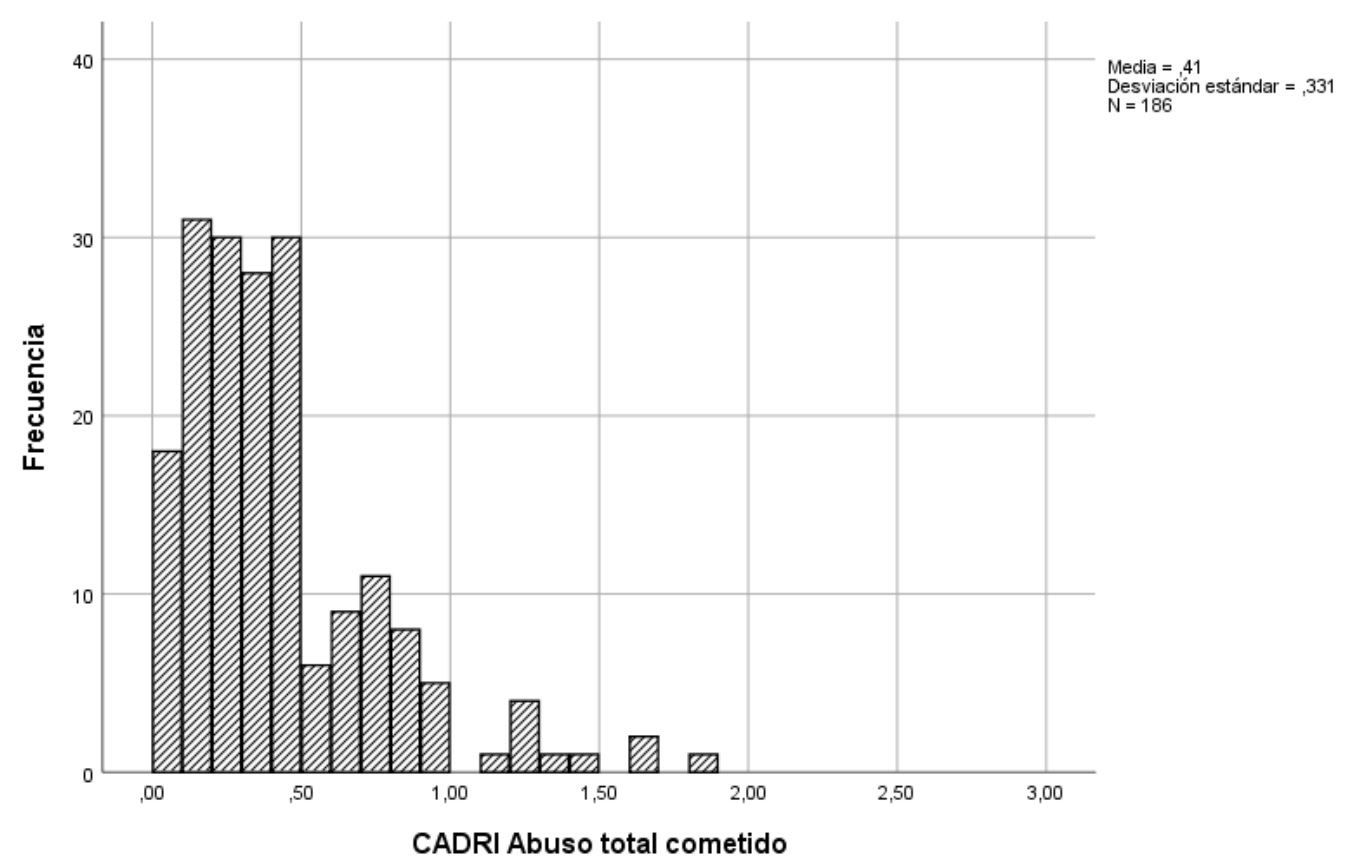

Gráfico 1. Distribución de valores de Abuso total cometido según CADRI y rango posible en abscisa.

En la Tabla 1 se exponen los estadísticos descriptivos para las subescalas y totales de la CADRI estratificados por género. El subtipo de violencia más cometido y sufrido fue el verbal emocional. Al examinar los resultados comparativamente entre sexos, se destaca la paridad (Tabla 1). La correlación entre violencia total cometida y sufrida fue $.77(p<.001)$. 
Tabla 1.

Violencia cometida y sufrida según subescalas y total de CADRI

\begin{tabular}{lccccccc}
\hline & \multicolumn{2}{c}{$\begin{array}{c}\text { Muestra total } \\
(N=186)(\mathrm{a})\end{array}$} & \multicolumn{2}{c}{$\begin{array}{c}\text { Mujeres } \\
(n=150)\end{array}$} & \multicolumn{3}{c}{$\begin{array}{c}\text { Varones } \\
(n=35)\end{array}$} \\
\hline \multicolumn{1}{c}{ Escala CADRI } & $M$ & $D E$ & $M$ & $D E$ & $M$ & $D E$ & $p$ \\
\hline Viol. verbal/emoc. cometida & 0.84 & 0.49 & 0.87 & 0.49 & 0.72 & 0.48 & .84 \\
Viol. verbal/emoc. sufrida & 0.86 & 0.56 & 0.84 & 0.55 & 0.95 & 0.58 & .86 \\
Amenazas cometidas & 0.18 & 0.31 & 0.18 & 0.30 & 0.20 & 0.35 & .18 \\
Amenazas sufridas & 0.23 & 0.41 & 0.21 & 0.36 & 0.34 & 0.58 & .23 \\
Violencia física cometida & 0.21 & 0.43 & 0.24 & 0.46 & 0.09 & 0.23 & .21 \\
Violencia física sufrida & 0.19 & 0.43 & 0.16 & 0.32 & 0.37 & 0.70 & .19 \\
\hline Abuso total cometido & 0.41 & 0.33 & 0.43 & 0.34 & 0.34 & 0.28 & .41 \\
\hline Abuso total sufrido & 0.43 & 0.39 & 0.40 & 0.34 & 0.55 & 0.57 & .43
\end{tabular}

Nota. CADRI: Conflict in Adolescents Dating Relationships Inventory. M: Media. DE: Desviación estándar. $p$ : significación asintótica bilateral de prueba de Mann Whitney, entre mujeres y varones. (a): En la estratificación por sexo hay un caso perdido por no tener consignada la categoría.

En cuando a los celos, medidos con la MJS, se halló que sólo los valores totales tuvieron una distribución normal (Kolmogorov-Smirnov $=0.063 ; p=.07$ ). Los valores de la subescala emocional tuvieron asimetría izquierda y la cognitiva y la conductual asimetría derecha.

En ambos sexos, la media de valores más alta se obtuvo con la subescala emocional (Tabla 2). La estratificación por sexo puso de manifiesto que no hubo diferencias estadísticamente significativas entre las medias.

Tabla 2.

Estadísticos descriptivos de valores MJS y comparación por sexo

\begin{tabular}{lccccccc}
\hline & \multicolumn{2}{c}{$\begin{array}{c}\text { Total } \\
(N=186)(\mathrm{a})\end{array}$} & \multicolumn{2}{c}{$\begin{array}{c}\text { Mujeres } \\
(n=150)\end{array}$} & \multicolumn{2}{c}{$\begin{array}{c}\text { Varones } \\
(n=35)\end{array}$} \\
\hline Escala & $M$ & $D E$ & $M$ & $D E$ & $M$ & $D E$ & $p$ \\
\hline MJS cognitiva & 22.06 & 10.88 & 21.77 & 10.75 & 23.37 & 11.61 & .50 \\
MJS emocional & 40.45 & 8.25 & 40.83 & 8.26 & 39.02 & 8.19 & .10 \\
MJS conductual & 15.87 & 9.33 & 15.84 & 8.97 & 16.17 & 10.93 & .99 \\
\hline MJS total & 78.38 & 18.99 & 78.45 & 19.25 & 78.55 & 18.18 & .97 \\
\hline
\end{tabular}

Nota. MJS: Multidimensional Jealousy Scale. M: Media. DE: Desviación estándar. p: significación asintótica bilateral de prueba de Mann Whitney. (a): En la estratificación por sexo hay un caso perdido por no tener consignada la categoría.

Respecto a las covariables, se obtuvo que las personas encuestadas informaron, en promedio, un estrés mediano durante la relación índex $(M=4.4 ; D E 2.6$; mín. 0; máx. 10) y haber sido un poco más fieles de lo que habían sido con ellos, sus previas parejas $(M=2.6 ; D E$ 3.1; mín. 0; máx. 10 vs. $M=3.8 ; D E 3.2$; mín. 0; máx. 10).

Al estratificar por sexo, se halló que la apreciación de estrés para la época de la pareja índex no tuvo diferencias significativas entre varones y mujeres $(M=4.5 ; M=4.4$ 
respectivamente; $p=.67)$. Las mujeres informaron en promedio haber sido menos infieles $(M=$ $2.47 ; M=3.14$ respectivamente; $p=.23)$ y haber sufrido más infidelidad $(M=3.89 ; M=3.18$ respectivamente; $p=.28$ ) aunque las diferencias tampoco fueron estadísticamente significativas.

El total de los participantes informó que no consumió, durante la relación de pareja índex, metanfetaminas, heroína ni inhalantes, pero el 1.6\% informó haber consumido alucinógenos, el $1 \%$ haber consumido cocaína y el 1\%, éxtasis. El $6.5 \%$ informó haber consumido marihuana algunos días al mes y el $3.3 \%$ haberla consumido, por lo menos, la mitad de los días del mes. El $32.8 \%$ informó no haber consumido alcohol; el 53.8\% haberlo consumido algunos días del mes y el 12.9\% haberlo consumido la mitad o más de los días del mes. El 17.2\%, informó haber consumido analgésicos sin prescripción médica y el $21 \%$ haber fumado tabaco durante la relación índex. Se computó la variable resumen "Sustancias consumidas", sumando las sustancias consumidas con la excepción del tabaco, y la media fue 1.48 ( $D E$ 1.9). Los varones tuvieron una media de $2(D E 2.5)$ y las mujeres $1.4(D E 1.7)$.

A continuación, se exponen los resultados de las correlaciones entre valores de CADRI total de abuso cometido y MJS, la edad al comienzo de la relación índex, el estrés, la valoración de infidelidad propia y de previas parejas y la variable sustancias consumidas (Tabla 3). La correlación entre el total de abuso cometido y MJS emocional fue la única que no alcanzó significación estadística. La correlación con la edad al comienzo en la relación índex fue negativa y las restantes positivas. Sobresalen las altas correlaciones con MJS conductual y con estrés (Tabla 3).

Tabla 3.

Correlaciones de CADRI abuso total perpetrado y variables independientes

\begin{tabular}{ll}
\hline \multicolumn{1}{c}{ Variable independiente } & $r$ \\
\hline MJS cognitiva & $.318^{* *}$ \\
MJS emocional & .029 \\
MJS conductual & $.502^{* *}$ \\
\hline MJS total & $.319^{* *}$ \\
\hline Sustancias consumidas & $.270^{* *}$ \\
Estrés & $.442^{* *}$ \\
Fidelidad del encuestado & $.280^{* *}$ \\
Fidelidad de previas parejas & $.223^{* *}$ \\
Edad al comienzo de la relación índex & $-.150^{*}$
\end{tabular}

Nota. $N=186$. MJS: Multidimensional Jealousy Scale. $r$ : Correlación Rho de Spearman. ${ }^{* *}$ correlación significativa en el nivel .01 (bilateral). *correlación significativa en el nivel .05 (bilateral).

A los efectos de considerar la variable CADRI abuso total cometido como variable dependiente en modelo multivariado y teniendo en consideración la asimetría de las distribuciones, se optó por formular modelo de regresión logística. Para ello, se dicotomizó su total en base a la mediana $(M d=0.3)$ y se consignó con el código 1 a los casos que tuvieron valor mayor que la mediana y con el código 0 a los que tuvieron igual o menor valor, y se les asignó las etiquetas "Grupo alta violencia" (47.8 \%) y "Grupo baja violencia" (52.2\%). Se formuló el modelo de regresión logística considerando variables independientes a todas aquellas de las que se informó la correlación en Tabla 3 y adicionando el sexo. La estimación se hizo mediante el método "condicional hacia adelante" con probabilidad de entrada de 0.05 y de salida 0.10 .

Las variables que quedaron en el modelo fueron MJS conductual ( $O R=1.09 ; p=.003$; $95 \%$ IC $1.03,1.2)$ y Estrés $(O R=1.41 ; p<.001 ; 95 \%$ IC $1.21,1.64)$. El modelo clasificó correctamente al $71.4 \%$ de los casos y ajustó adecuadamente a los datos (Hosmer y Lemeshow= 
$8.11(\mathrm{gl}) \mathrm{p}=.42)$. Las variables en el modelo explican no menos del $22 \%$ de la varianza $(R$ cuadrado de Cox y Snell=0.22; $R$ cuadrado de Nagelkerke=0.30).

\section{Discusión}

Este estudio tuvo los objetivos de describir las manifestaciones violentas en una muestra de estudiantes universitarios de la Universidad de Cuenca y de examinar sus correlatos con los celos y otros factores teóricamente relevantes. Los datos se obtuvieron con una encuesta que requería a los encuestados informar sobre las variables en el contexto de una relación de pareja amorosa intensa, es decir que hubiera movilizado sus afectos, el romanticismo o el amor según sus propias interpretaciones de lo que ello implica. Los estudiantes respondieron sobre parejas con considerable duración y que, en casi la mitad de los casos, aún continuaban unidas. Esas parejas fueron iniciadas, en promedio, a los 18 años, por lo tanto, resultan relativamente alejadas de la época en la que suelen darse los primeros contactos adolescentes. Ambas características tienden a aportar confiabilidad a las referencias pues se trata de memorias con menos afectación del transcurso del tiempo y, coincidentemente con lo pretendido en la consigna, son memorias de experiencias significativas. Por lo tanto, dado que la información buscada era vulnerable al sesgo de memoria, cabe sostener que tal riesgo fue minimizado.

Desde un punto de vista valorativo cualquier expresión de violencia es indeseable, pero resulta particularmente preocupante aquella población que exterioriza la violencia más frecuente o intensamente. Considerando el perfil general de VPEU obtenido con CADRI, se destaca la tendencia general a los valores bajos y al predominio de la violencia verbal/emocional, de manera homóloga a lo hallado en reciente estudio latinoamericano (Delucchi et al., 2020). Sin embargo, cabe destacar que una proporción de los estudiantes de la muestra algo mayor que el 5 $\%$ alcanzó valores preocupantes y que el $60.2 \%$ informó haber cometido por lo menos un acto violento físico durante la relación índex.

Otro hallazgo digno de discusión es que hubo paridad entre los valores de violencia cometida por el grupo femenino y el grupo masculino. Previos estudios informaron sobre la bilateralidad de las agresiones en los noviazgos juveniles (Chan et al., 2008; Malik et al., 1997; Straus \& Ramirez, 2007; Taylor \& Mumford, 2016) e, incluso, sobre mayores valores en el grupo femenino (Arbach-Lucioni et al., 2015; Elmquist et al., 2016). Si bien en este estudio no se exploraron los subtipos determinados por la unidireccionalidad agresiva -sólo desde el varón a la mujer o sólo desde la mujer al varón- y por la bidireccionalidad (Straus \& Gozjolko, 2014), los hallazgos están en línea con el planteo de la predominante reciprocidad de la violencia en las parejas de los jóvenes. En el presente estudio, además de la paridad, se verificó que la violencia cometida varió concomitantemente con la violencia sufrida. El hallazgo también orienta a sostener que en las parejas se produjeron interacciones violentas bilaterales pues el contexto para ambos tipos de violencia era la misma pareja índex. Interesantemente, Fernández-Fuertes, Fuertes y Pulido (2006) también obtuvieron altas correlaciones entre violencia cometida y sufrida y hacen una interpretación equivalente, señalando la posible existencia de patrones de violencia mutua. Este aspecto de la VPEU podría estar relacionado con los hábitos de conducta y expresividad compartidos $\mathrm{y}$, también, con la predisposición a la acción, ya sea defensiva $\mathrm{u}$ ofensiva, que tienen los jóvenes. Coincidentemente con Taylor y Munford (2016), se considera que el hallazgo orienta a sostener que los servicios no deberían contemplar grupos monolíticamente separados en víctimas y en victimarios.

Sean los valores de violencia informados por las mujeres similares o mayores que los informados por los varones, la interpretación de ninguna manera atenúa la internacional e histórica regularidad del mayor impacto de la violencia masculina, pero pone de manifiesto un aspecto digno de atención: los comportamientos violentos en parejas de este grupo etario 
trascienden, por lo menos, a los sexos biológicos. Ese aspecto es relevante no sólo a los efectos preventivos, sino, también, para las tareas periciales. Estas evidencias imponen al experto, al momento de evaluar el caso específico, la precaución de explorar la existencia de eventual estilo interaccional violento.

Los hallazgos orientan a sostener que ofrecer servicios preventivos específicos a la población estudiantil universitaria local está justificado no sólo moralmente sino, también, empíricamente. También aduna en este sentido la contrastación con estudios de otras latitudes. Los valores medios fueron mayores en esta muestra que los del grupo etario más parecido -19 años- de una muestra española (Fernández-Fuertes et al., 2006) y que los de la muestra argentina (Delucchi et al., 2020). Estas consideraciones dejan planteada la necesidad de especial atención preventiva que tienen los estudiantes locales y, también, interrogantes para futuros estudios, pues será importante explorar la eventual influencia de factores de nivel macro como la inequidad de género (Okeke, Mumford, \& Rothman, 2019) o de pautas culturales relacionadas con el temprano comienzo de la relaciones románticas.

Previamente a discutir acerca de la asociación de los factores contemplados en este estudio con la VPEU, será conveniente exponer algunos hallazgos descriptivos. En cuanto a los celos totales, se encontró una distribución normal, pero entre los celos emocionales por una parte y los celos cognitivos y comportamentales por la otra, se encontró marcado contraste. Los estudiantes en general informaron que sus celos fueron predominantemente emocionales. Las diferencias entre mujeres y varones no fueron significativas, a diferencia de lo que fuera informado de Argentina, donde se encontró que las mujeres informaban significativamente mayor quantum de celos que los varones (Delucchi et al., 2020).

De los hallazgos sobre la apreciación del propio estrés, la valoración de la infidelidad propia y de la de las previas parejas, resulta destacable que se informara el padecimiento de un grado de estrés mediano y que no tuviera diferencias significativas entre sexos.

Del perfil del consumo de sustancias durante la pareja índex, se destaca que casi el $10 \%$ informó haber consumido marihuana y que dos tercios consumieron alcohol. Esta información pone de manifiesto un consumo menor de esas sustancias que en la población estudiantil de La Plata (Delucchi et al., 2020), si bien no deja de ser una información que debería ser foco de futuros estudios, pues la región de la sierra ecuatoriana está considerada como una de las que tiene mayor consumo de alcohol en la población juvenil (Instituto Nacional de Estadisticas y Censos, 2014).

Exceptuando los celos emocionales, la relación entre cada una de las demás covariables con la VPEU resultó estadísticamente significativa. Interesantemente, se verificó que, a diferencia de lo hallado con las restantes medidas, a menor edad al comienzo de la relación de pareja correspondió mayor valor de VPEU. El hallazgo es análogo a lo informado en estudios foráneos (Delucchi et al., 2020; Johnson et al., 2014), si bien en éstos últimos la correlación fue mayor. El signo negativo de esa asociación se mantuvo al formular el modelo multivariado, pero dejó de ser estadísticamente significativa, al igual que la asociación de otros factores. Solamente se mantuvieron con significación estadística, las asociaciones de los celos comportamentales y del estrés con la condición de pertenecer al grupo de alta violencia. Si bien el diseño del estudio no permite expedirse respecto a causalidad, los hallazgos con compatibles con un modelo explicativo multifactorial en el que los celos más exteriorizados y el estrés influyen, finalmente, en los comportamientos violentos en la pareja. La asociación entre estos factores y la VPEU de ninguna manera da cuenta de la variancia completa del fenómeno. Investigaciones futuras podrán identificar otros factores influyentes, pero, mientras tanto, el hallazgo permite disponer de un blanco empíricamente delimitado para orientar las acciones preventivas. Tanto la exteriorización comportamental de los celos como el estrés concomitante resultan factores pasibles de intervención generando una perspectiva optimista respecto a las posibilidades de disminuir la violencia íntima. 
Además de las limitaciones del diseño transversal, en este estudio se reconocen otras limitaciones. Los resultados no pueden generalizarse a los estudiantes de la universidad; si bien la muestra abarca la amplia mayoría de los estudiantes de dos carreras (186 de 220 elegibles) no es de ninguna manera una muestra representativa del universo estudiantil local. La marcada desproporción entre mujeres y varones y eventuales peculiaridades de los estudiantes de esas carreras podrían estar sesgando los resultados.

Con los datos obtenidos no se puede establecer si el consumo sustancias fue posterior a la violencia, y consumido como acción paliativa del disconfort, o previo, de manera tal que eventuales efectos psicoactivos pudieran estar vigentes al momento de la interacción violenta. Tampoco si se trata de un consumo que sólo causó los efectos transitorios o si alcanzó a conformar un trastorno perdurable. Este factor debería continuar siendo investigado y, por lo menos teóricamente, debería también ser considerado entre las acciones preventivas. No puede desestimarse su eventual influencia perniciosa pues el consumo de sustancias tiene complejas y bidireccionales relaciones con la violencia (Levitt \& Cooper, 2010). El consumo puede intervenir directamente como factor desinhibidor y de manera indirecta cuando, consumido paliativamente, posterga la mejor resolución de los conflictos o perturba los vínculos.

Si bien, arriba se comentaron características del estudio que atenuaron el riesgo de los sesgos de memoria y de deseabilidad social, tales como la contextualización a una relación amorosamente significativa y el anonimato, no puede dejar de reconocerse la posibilidad de algún grado de influencia en la información obtenida.

Por último, cabe destacar que el estudio es, hasta donde se pudo corroborar con las bases bibliográficas indexadas, el primer intento empírico de relacionar los celos con la violencia en las parejas de jóvenes estudiantes en Ecuador. Los factores que se asociaron con la VPEU son pasibles de intervención y deberían ser considerados a la hora de diseñar programas preventivos basados en evidencias. La necesidad de esos programas ya ha sido resaltada en otros países (Khubchandani et al., 2017) y es esperable que este estudio contribuya a que sea reconocida y satisfecha localmente.

\section{Referencias}

American Psychiatric Association. (2013). Cross-cutting level 2: Substance use - adult. Retrieved from http://www.psychiatry.org/File

Arbach-Lucioni, K., Nguyen-Vo, T., \& Bobbio, A. (2015). Violencia física en el noviazgo: análisis de los tipos diádicos en población argentina. Revista Argentina de Ciencias Del Comportamiento, 7(2), 38-46.

Aula de Derechos Humanos. (2018). Protocolo de prevención y actualización en casos de acoso, discriminación y violencia basada en género y orientación sexual en la Universidad de Cuenca. Cuenca, Ecuador: Universidad de Cuenca.

Black, M. C., Basile, K. C., Breiding, M. J., Smith, S. G., Walters, M. L., Merrick, M. T., ... Stevens, M. R. (2010). National Intimate Partner and Sexual Violence Survey 2010. Summary Report. Atlanta, GA: National Center for Injury Prevention and Control, Centers for Disease Control and Prevention.

Brem, M. J., Shorey, R. C., Rothman, E. F., Temple, J. R., \& Stuart, G. L. (2018). Trait Jealousy Moderates the Relationship Between Alcohol Problems and Intimate Partner Violence Among Men in Batterer Intervention Programs. Violence Against Women, 24(10), 11321148. doi: $10.1177 / 1077801218781948$

Burchell, J. L., \& Ward, J. (2011). Sex drive, attachment style, relationship status and previous infidelity as predictors of sex differences in romantic jealousy. Personality \& Individual Differences, 51(5), 657-661. doi: 10.0.3.248/j.paid.2011.06.002 
Burk, W. J., \& Seiffge-Krenke, I. (2015). One-sided and mutually aggressive couples: Differences in attachment, conflict prevalence, and coping. Child Abuse \& Neglect, 50, 254266. doi: 10.1016/j.chiabu.2015.08.011

Buunk, A. P., \& Massar, K. (2019). Intimate Partner Violence in Nicaragua: The Role of Possessive Jealousy, Intrasexual Competitiveness, Life History, Mate Value, and Stress. Journal of Interpersonal Violence. doi: 10.1177/0886260519842854

Capaldi, D. M., Knoble, N. B., Shortt, J. W., \& Kim, H. K. (2012). A Systematic Review of Risk Factors for Intimate Partner Violencie. Partner Abuse, 3(2), 231-280. doi: 10.1891/19466560.3.2.231.A

Cascardi, M., Avery-Leaf, S., \& O'Leary, K. D. (1994). Building a gender sensitive model to explain male and female use of dating violence: Preliminary results. Los Angeles, CA: Paper presented at the 102nd annual Meeting of the American Psychological Association.

Center for Disease Control and Prevention. (2000). Youth risk behavior surveillance--United States 1999. Morbidity and Mortality Weekly Report, 49(5), 1-96.

Chang, D. F., Shen, B. J., \& Takeuchi, D. T. (2009). Prevalence and demographic correlates of intimate partner violence in Asian Americans. International Journal of Law and Psychiatry, 32(3), 167-175. doi: 10.1016/j.ijlp.2009.02.004

Chan, K. L., Straus, M. A., Brownridge, D. A., Tiwari, A., \& Leung, W. C. (2008). Prevalence of dating partner violence and suicidal ideation among male and female university students worldwide. Journal of Midwifery \& Women's Health, 53(6), 529-537. doi: 10.1016/j.jmwh.2008.04.016

Consejo Universitario. (2018). Reglamento de la Unidad de Bienestar Universitario de la Universidad de Cuenca. Cuenca, Ecuador: Universidad de Cuenca.

Costa, A. L., Sophia, E. C., Sanches, C., Tavares, H., \& Zilberman, M. L. (2015). Pathological jealousy: Romantic relationship characteristics, emotional and personality aspects, and social adjustment. Journal of Affective Disorders, 174, 38-44. doi: 10.1016/j.jad.2014.11.017

Daly, M., Wilson, M., \& Weghorst, S. J. (1982). Male sexual jealousy. Ethology and Sociobiology, 3(1), 11-27. doi: 10.1016/0162-3095(82)90027-9

Davis, K. E., Ace, A., \& Andra, M. (2000). Stalking perpetrators and psychological maltreatment of partners: Anger-jealousy, attachment insecurity, need for control, and break-up context. Violence and Victims, 15(4), 407-426. doi: 10.1891/0886-6708.15.4.407

Delucchi, G., Leon-Mayer, E., \& Folino, J. (2018). Celos y violencia en parejas de estudiantes universitarios. Vertex, XXIX, 245-254.

Delucchi, G., Leon-Mayer, E., \& Folino, J. O. (2020). Intimate partner violence and jealousy in Argentinian university students. Violence and Gender, submitted paper.

DeSteno, D., Valdesolo, P., \& Bartlett, M. Y. (2006). Jealousy and the threatened self: Getting to the heart of the green-eyed monster. Journal of Personality and Social Psychology, 91(4), 626-641. doi: 10.1037/0022-3514.91.4.626

DiBello, A. M., Rodriguez, L. M., Hadden, B. W., \& Neighbors, C. (2015). The green eyed monster in the bottle: Relationship contingent self-esteem, romantic jealousy, and alcoholrelated problems. Addictive Behaviors, 49, 52-58. doi: 10.1016/j.addbeh.2015.05.008

do Nascimento Paixao, G. P., Gomes, N. P., Freire Diniz, N. M., Menezes Couto, T., Carneiro Vianna, L. A., \& Pessoa dos Santos, S. M. (2014). Situations which precipitate conflicts in the conjugal relationship: the women's discourse. Text Context Nursing, 23(4), 1041-1049.

Douglas, K. S., Hart, S. D., Webster, C. D., \& Belfrage, H. (2013). HCR-20 v3 Assessing Risk for Violence. Vancouver: Mental Health, Law, and Policy Institute, Simon Fraser University.

Elmquist, J., Wolford-Clevenger, C., Zapor, H., Febres, J., Shorey, R. C., Hamel, J., \& Stuart, G. L. (2016). A gender comparison of motivations for physical dating violence among college 
students. Journal of Interpersonal Violence, 31(1). doi: 10.1177/0886260514555130

Elphinston, R., Feeney, J., \& Noller, P. (2011). Measuring romantic jealousy: Validation of the multidimensional jealousy scale in Australian samples. Australian Journal of Psychology, 63(4), 243-251.

Fernández-Fuertes, A. A., Fuertes Martín, J. A., \& Fernández Pulido, R. (2006). Evaluación de la violencia en las relaciones de pareja de los adolescentes . Validación del Conflict in Adolescent Dating Relationships Inventory. International Journal of Clinical and Health Psychology, 6(2), 339-358. doi: 10.1017/CBO9781107415324.004

Giordano, P. C., Soto, D. A., Manning, W. D., \& Longmore, M. A. (2010). The characteristics of romantic relationships associated with teen dating violence. Social Science Research, 39(6), 863-874. doi: 10.1016/j.ssresearch.2010.03.009

Goldenberg, T., Jadwin-cakmak, L., \& Harper, G. W. (2018). Intimate Partner Violence Among Transgender Youth: Associations with Intrapersonal and Structural Factors. Violence and Gender, 5(1), 19-25. doi: 10.1089/vio.2017.0041

Gomez, J., Orchowski, L., Pearlman, D. N., \& Zlotnick, C. (2019). Alcohol Use and Intimate Partner Violence among Community College Women: An Exploration of Protective Factors. Violence and Gender, 6(3), 187-195. doi: 10.1089/vio.2018.0023

Greer, B., Taylor, R. W., Cella, M., Stott, R., \& Wykes, T. (2020). The contribution of dynamic risk factors in predicting aggression: A systematic review including inpatient forensic and non-forensic mental health services. Aggression and Violent Behavior, 53(April), 101433. doi: 10.1016/j.avb.2020.101433

Haas Bueno, J. F., \& Carvalho, L. de F. (2005). Um Estudo de Revisão do Inventário de Ciúme Romântico ( ICR ). Psicologia: Reflexão e Crítica, 25(3), 435-444.

Ihongbe, T. O., \& Masho, S. W. (2018). Gender Differences in the Association Between Synthetic Cannabinoid Use and Teen Dating Violence Victimization. Violence and Gender, 3(00), 1-7. doi: 10.1089/vio.2017.0046

Instituto Nacional de Estadisticas y Censos. (2014). Encuesta Nacional de Salud y Nutrición. Quito: Dirección de Innovación en Métricas y Metodologías.

Instituto Nacional de Estadísticas y Censos. (2019). Encuesta nacional sobre relaciones familiares y violencia de género contra las mujeres (ENVIGMU). Quito.

Johnson, W. L., Giordano, P. C., Manning, W. D., \& Longmore, M. (2014). The Age-IPV Curve: Changes in the Perpetration of Intimate Partner Violence During Adolescence and Young Adulthood. Journal of Youth and Adolescence, 44(3), 708-726. doi: 10.1007/s10964-014-0158-Z

Khubchandani, J., Clark, J., Wiblishauser, M., Thompson, A., Whaley, C., Clark, R., \& Davis, J. (2017). Preventing and Responding to Teen Dating Violence : Violence and Gender, 4(4), 144-151. doi: 10.1089/vio.2017.0043

Kwan, J., Sparrow, K., Facer-Irwin, E., Thandi, G., Fear, N. T., \& MacManus, D. (2020). Prevalence of intimate partner violence perpetration among military populations: A systematic review and meta-analysis. Aggression and Violent Behavior, 53(April), 101419. doi: 10.1016/j.avb.2020.101419

Levitt, A., \& Cooper, M. L. (2010). Daily alcohol use and romantic relationship functioning: evidence of bidirectional, gender-, and context-specific effects. Personality \& Social Psychology Bulletin, 36(12), 1706-1722. doi: 10.1177/0146167210388420

Lucas, C., Pereira, H., \& Esgalhado, G. (2012). Evaluation of Romantic Jealousy: Psychometric Study of the Multidimensional Jealousy Scale for the Portuguese Population. Psychology, Community \& Health, 1(2), 151-162. doi: 10.5964/pch.v1i2.26

Malik, S., Sorenson, S. B., \& Aneshensel, C. S. (1997). Community and dating violence among adolescents: Perpetration and victimization. Journal of Adolescent Health, 21(5), 291-302. doi: 10.1016/S1054-139X(97)00143-2 
Miller, S. L., \& Maner, J. K. (2008). Coping with Romantic Betrayal: Sex Differences in Responses to Partner Infidelity. Evolutionary Psychology, 6(3), 413-426. doi: 10.1177/147470490800600305

Mostardinha, A. R., Bártolo, A., Bonifácio, J., \& Pereira, A. (2019). Validation of the alcohol, smoking and substance involvement screening test (ASSIST) among university students. Acta Medica Portuguesa, 32(4), 279-288. doi: 10.20344/amp.10650

Muñoz-Rivas, M. J., Graña, J. L., O’Leary, K. D., \& González, M. P. (2007). Aggression in Adolescent Dating Relationships: Prevalence, Justification, and Health Consequences. Journal of Adolescent Health, 40(4), 298-304. doi: 10.1016/j.jadohealth.2006.11.137

Murphy, A. M., \& Russell, G. (2016). Rejection Sensitivity, Jealousy, and the Relationship to Interpersonal Aggression. Journal of Interpersonal Violence, 33(13), 2118-1229. doi: $10.1177 / 0886260515622572$

Okeke, N. L., Mumford, E. A., \& Rothman, E. F. (2019). Adolescent Relationship Aggression Perpetration and Victimization in the Context of Neighborhood Gender Equality. Violence and Gender, 6(2), 131-138. doi: 10.1089/vio.2018.0028

Organización Mundial de la Salud. (2011). La prueba de detección de consumo de alcohol, tabaco y sustancias (ASSIST). Oms. Whashington, D.C.: Organización Panamericana de la Salud.

Organización Panamericana de la Salud. (2003). Informe mundial sobre la violencia y la salud. Washington: Organización Panamericana de la Salud, Oficina Regional para las Américas de la Organización Mundial de la Salud.

Paixão, G. P. do N., Gomes, N. P., Diniz, N. M. F., Couto, T. M., Vianna, L. A. C., \& Santos, S. M. P. dos. (2014). Situations which precipitate conflicts in the conjugal relationship: the women's discourse. Texto \& Contexto - Enfermagem, 23(4), 1041-1049. doi: 10.1590/010407072014003290013

Pfeiffer, S. M; Wong, P. T. (1989). Multidimensional Jealousy. Journal of Social and Personal Relationships, 6, 181-196.

Pines, A., \& Aronson, E. (1983). Antecedents, correlates, and consequences of sexual jealousy. Journal of Personality, 51(1), 108-136. doi: 10.1111/j.1467-6494.1983.tb00857.x

Prinstein, M., Boegers, J., \& Vernberg, E. (2001). Overt and relational aggression in adolescents: Social-psychological adjustments of aggresors and victims. Journal of Clinical Child Psychology, 30(4), 479-491.

Roscoe, B., \& Callahan, J. (1985). Adolescents' self- report of violence in families and dating relations. Adolescence, 20, 545-554.

Salmivalli, C., \& Kaukiainen, A. (2004). Female aggression revisited. Variable- and personcentered approaches to studying gender differences in different types of aggressive behavior. Aggresive Behavior, 30, 158-163.

Seiffge-Krenke, I., \& Burk, W. J. (2015). "Bad Romance": Links between Psychological and Physical Aggression and Relationship Functioning in Adolescent Couples. Behavioral Sciences (2076-328X), 5(2), 305-323. doi: 10.0.13.62/bs5020305

Straus, M. A., \& Gozjolko, K. L. (2014). "Intimate Terrorism" and Gender Differences in Injury of Dating Partners by Male and Female University Students. Journal of Family Violence, 29(1), 51-65. doi: 10.1007/s10896-013-9560-7

Straus, M. A., \& Ramirez, I. L. (2007). Gender symmetry in prevalence, severity, and chronicity of physical aggression against dating partners by university students in Mexico and USA. Aggressive Behavior, 33(4), 281-290. doi: 10.1002/ab.20199

Swanson, J. W., Holzer, C. E., Ganju, V. K., \& Jono, R. T. (1990). Violence and psychiatric disorder in the community: Evidence from the Epidemiologic Catchment Area surveys. HOSP-COMMUNITY-PSYCHIATRY, (41), 761-770.

Swanson, J. W., Monahan, J., \& Steadman, H. (1994). Mental Disorder, Substance Abuse and 
Community Violence: An Epidemiological Approach. En J. Monahan \& H. Steadman (Eds.), Violence and Mental Disorder - Developments in Risk Assessment (pp. 101-136). Chicago: University of Chicago Press.

Tani, F., \& Ponti, L. (2016). The Romantic Jealousy as Multidimensional Construct: A Study on the Italian Short Form of the Multidimensional Jealousy Scale. The Open Psychology Journal, 09(1), 111-120. doi: 10.2174/1874350101609010111

Taylor, B. G., \& Mumford, E. A. (2016). A National Descriptive Portrait of Adolescent Relationship Abuse: Results From the National Survey on Teen Relationships and Intimate Violence. Journal of Interpersonal Violence, 31(6), 963-988. doi: 10.1177/0886260514564070

Temple, J. R., Ph, D., Choi, H. J., Ph, D., Elmquist, J., A, M., .. A, M. (2016). Psychological Abuse , Mental Health , and Acceptance of Dating Violence Among Adolescents. Journal of Adolescent Health, 59(2), 197-202. doi: 10.1016/j.jadohealth.2016.03.034

Wolfe, D. A. (2001). Conflict in Adolescent Dating Relationships Inventory. Los Altos, CA: Sociometrics Corporation.

Wolfe, D. A., Scott, K., Reitzel-Jaffe, D., Wekerle, C., Grasley, C., \& Straatman, A.-L. (2001). Development and validation of the Conflict in Adolescent Dating Relationships Inventory. Psychological Assessment, 13(2), 277-293.

Contribución de los autores: a) Concepción y diseño del trabajo; b) Adquisición de datos; c) Análisis e interpretación de datos; d) Redacción del manuscrito; e) revisión crítica del manuscrito.

X.G.V. ha contribuido en a,b,c,d,e; J.O.B. en a,b,c,d; G.D. en a,c,d,e; E.L.M. en a,c,d,e; J.F. en a,c,d,e.

Editora científica responsable: Dra. Cecilia Cracco 